\title{
Respuestas
}

Año 17

No. 2

Diciembre 2012

ISSN 0122-820X

\section{Desarrollo de un módulo didáctico para control angular de un péndulo suspendido}

\author{
Moreno G. Francisco. E. ${ }^{1} \quad$ Becerra V. José A. ${ }^{2} \quad$ Ayala G. Yulianny E. ${ }^{3} \quad$ Alarcón Ricardo F.
}

Recibido:

Junio 23 de 2012

Aceptado:

Noviembre 12 de 2012

\section{Resumen}

Este trabajo presenta el desarrollo de un módulo didáctico con la implementación de un controlador proporcional - integrativo (PI) en un péndulo suspendido, donde se regula la velocidad de la hélice de forma se obtenga el ángulo deseado y se mantenga estable ante la presencia de perturbaciones adicionadas al péndulo. Además el módulo didáctico, le fue desarrollado un sistema de adquisición de datos con un microcontrolador atmega atmel 328 en comunicación serial con la plataforma matlab $®$ simulink $®$, la cual permite un monitoreamiento en línea en tiempo real del comportamiento del péndulo para sus posteriores análisis y prácticas a los estudiantes en el área de teoría control e ingeniería. El comportamiento del sistema fue satisfactorio, se alcanzaron respuestas transitorias no mayores a cuatro segundos por parte del controlador PI para estabilizar el sistema ante la presencia de perturbaciones o cambios tipo escalonado en su setpoint.

Palabras clave: péndulo, controlador PI.

\section{Abstract}

This paper presents development of a training module to implement a proportional - integrative controller (PI) of a suspended pendulum which regulates the speed of the propeller to obtain the desired angle and to remain stable in the presence of disturbances added into a pendulum. Besides the training module was developed a data acquisition system with a microcontroller atmel atmega 328 serial communication with matlab ${ }^{\circledR}$ simulink $\circledR$ platform, which enables real-time online supervisory behavior for subsequent analysis pendulum and students practice in the area of control and engineering. The system performance was satisfactory transient responses were achieved no more than four seconds by the PI controller to stabilize the system in the presence of disturbances or changes in its setpoint step type.

Keywords: Pendulum, PI controller, system.

${ }^{3}$ Ingeniero Electrónico, Grupo de Investigación en Automatización

y Control - GIAC, Universidad Francisco de Paula Santander, Cúcuta - Colombia. Correo electrónico: yulielectronica@hotmail.com 


\section{Introducción}

L a limitación de sistemas didácticos económicos a la comunidad científica académica, presenta un reto a solucionar en todas las disciplinas con apoyo de herramientas electrónicas y mecánicas al alcance de dicha comunidad. Este hecho ha motivado a las universidades a disponer de laboratorios polivalentes que puedan ayudar a los estudiantes a desarrollarse y capacitarse en los diferentes campos de la ingeniería [1]. Hoy en día, la finalidad y el reto que se tiene en las universidades es ir a la vanguardia en innovación práctica, a partir de la creación de herramientas que faciliten el aprendizaje en la formación de profesionales más competitivos. Por lo tanto, el desarrollo de módulos didácticos contempla beneficios y perspectivas a ser alcanzados, permitiéndose al futuro profesional aprender y afrontar los diversos proyectos a nivel de ingeniería [1].

Un péndulo simple es un sistema físico que exhibe un movimiento mecánico. Este consiste en una masa adherida a una barra de longitud fija y suspendida en un punto de equilibrio. El movimiento ocurre en el plano vertical comandado por la fuerza gravitacional [2]. Los péndulos son conocidos como buenos resonadores y adecuados para el estudio de periodos largos de estabilidad [3], punto importante para el área del control.

Dentro de esa área de control, su filosofía parte de una estructura de control realimentado que tiene como objetivo lograr ciertas propiedades deseables. La viabilidad del control en campos donde hay cambios en los parámetros de sistemas y la presencia de perturbaciones, tal es el caso de péndulos, hace que sea un reto, de forma que se proporcione un controlador robusto para sistemas dinámicos con acciones de control que lleve al sistema a operar en regiones estables, [4][5][6].

Desde el punto de vista pedagógico y a diferentes tecnologías, se han desarrollado en los últimos años, tal es el caso de [7], donde a partir de un microcontrolador PICF684 implementaron un controlador PID para estabilizar un sistema inherentemente inestable, dicho sistema fue un péndulo invertido controlado en una plataforma de $360^{\circ}$ libres rotacional. Experiencias para este tipo de proyectos, por ejemplo $[8,13]$, dichos autores desarrollaron un sistema de balanceamiento de la base de un péndulo en el eje horizontal a través de un controlador PD, sin embargo, el éxito de dicha técnica dependió de algunas limitaciones relacionadas a vibraciones, torques y rozamientos especialmente en su sistema de desplazamiento, pero de cualquier forma su estructura de control presentó robustez.

Este trabajo esta desarrollado de la siguiente manera: primeramente es propuesto un modelo matemático del péndulo suspendido donde se obtuvo la función de transferencia teóricamente. Después desde el punto de vista experimental, se prosigue con la identificación del modelo experimental a partir de mediciones de entrada/salida. Por último fue propuesto e implementado una estructura de control en matlab / simulink tiempo real al módulo didáctico de péndulo suspendido para respectivas investigaciones.

\section{Modelamiento e identificación del sistema péndulo suspendido}

El diagrama esquemático e imagen del módulo didáctico péndulo suspendido puede ser observado en la Figura 1. Es claro que el orden del análisis y control del sistema físico, depende necesariamente de conocer el modelo (función de transferencia) de este mismo. En este sistema péndulo, su actuador es un motor corriente directa (DC) con una hélice en un extremo del péndulo, de forma que proporcione una fuerza empuje correctiva $T(s)$, para que el ángulo $\theta(s)$ que está entre el brazo oscilante y el eje vertical sea la variable deseada (setpoint) a contralar. 


\section{Respuestas}

Año 17

No. 2

Diciembre 2012 ISSN 0122-820X
Figura 1. Módulo didáctico péndulo suspendido

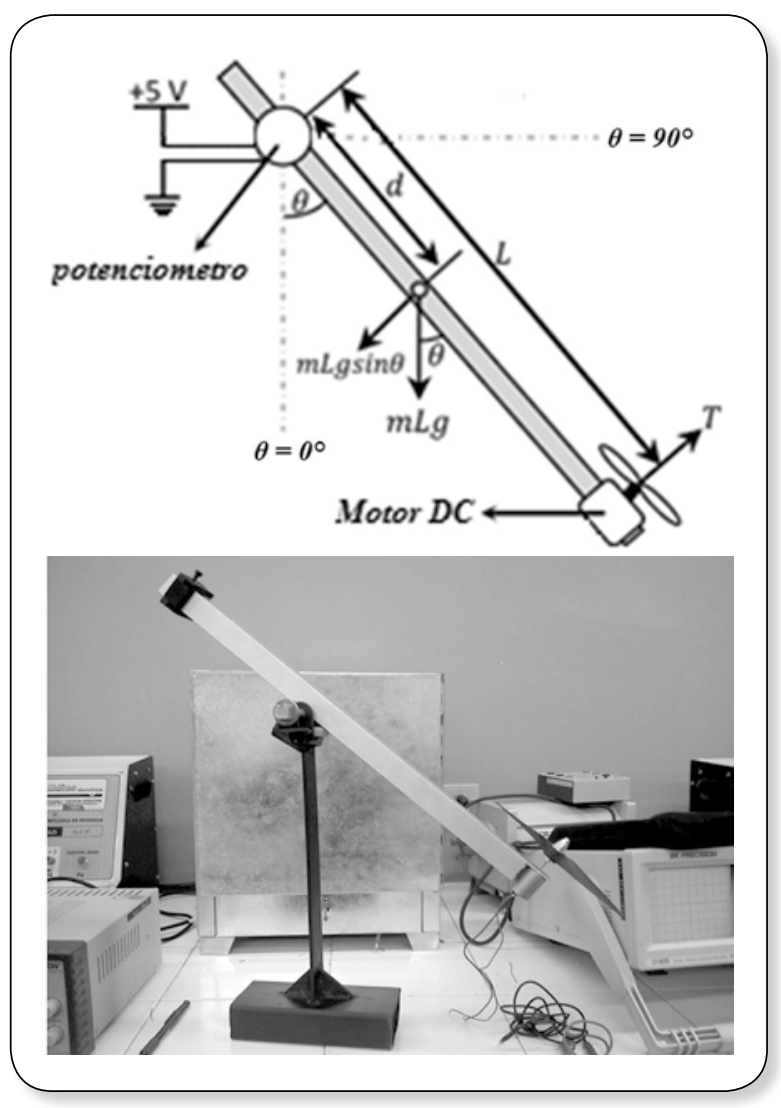

Desde la figura 1 se puede escribir la ecuación (1) que representa la función de transferencia en términos de Laplace ángulo del péndulo suspendido con respecto a la fuerza empuje correctiva, dicha función esta basada en el aporte de [4] la cual presentaron un modelo no lineal, para luego ser aproximado a una función lineal como estrategia de simplificación en control lineal.

$$
\frac{\theta(s)}{T(s)}=\frac{1}{J s^{2}+c s+m_{L} \mathrm{~g} d}
$$

\section{Donde:}

$T(s)=$ Fuerza de empuje producida por el motor DC.

$L=$ Longitud brazo péndulo

$m=$ Masa del péndulo

$m_{\mathrm{L}}=$ Masa del brazo del péndulo

$d^{L}=$ La distancia desde el punto de suspensión a centro de masas.

$\mathrm{J}=$ Momento de inercia.

$g \quad=$ Gravedad

c $=$ Coeficiente de amortiguamiento.
La relación entre el voltaje $V(s)$ aplicado al motor DC con respecto a la fuerza de empuje $T(s)$ puede relacionarse a través de la siguiente ecuación:

$$
T(s)=K_{m} \cdot V(s)
$$

Donde la constante $K_{m}$ puede ser definida como la ganancia que se necesita para obtener el mínimo ángulo en situación estable $\theta$ ss con respecto a un voltaje $V$ aplicado,

$$
K_{m}=\frac{m_{L} g d \sin \theta_{s s}}{V}
$$

A partir de las ecuaciones (1) y (2), podemos restructurar la función de transferencia del sistema péndulo suspendido en termino $\theta(s) /$ $V(s)$ como se presenta en la ecuación (4)

$$
\frac{\theta(s)}{V(s)}=\frac{K_{m} / J}{s^{2}+\frac{c}{J} s+\frac{m_{L} \mathrm{~g} d}{J}}
$$

A partir de las técnicas para el cálculo de los parámetros dinámicos de este tipo de sistemas de control [8,9], fue calculado para dicho sistema una frecuencia natural $w_{n}=1.047 \mathrm{rad} / \mathrm{s}$ y un factor de amortiguamiento de $\zeta=0.8$. La ecuación característica relacionada a la función de transferencia de la ecuación (4) fue igualada a un polinomio característico de segundo orden, de la siguiente forma.

$$
s^{2}+\frac{c}{J} s+\frac{m_{L} \mathrm{~g} d}{J}=s^{2}+2 \zeta w_{n} s+w_{n}{ }^{2}
$$

Si los parámetros físicos son sustituidos en la igualdad, ecuación (5),

tales como

$m_{L}=0.36 \mathrm{Kg}, d=0.08 \mathrm{~m}, g=9.8 \mathrm{~m} / \mathrm{s}^{2}$,

puede obtenerse el momento de inercia $J=0.257 \mathrm{Kgm}^{2}$

y coeficiente de amortiguamiento $c=0.43 \mathrm{Nms} / \mathrm{rad}$. 
Considerando que el Sin $\theta_{s s} \approx \theta_{s}$ debido al hecho de que el voltaje de alimentación y el ángulo deseado se conocen, entonces la constante $K_{m}=0.16$ de la ecuación (4). Finalmente la función de transferencia $\theta(s) / \mathrm{V}(s)$ del péndulo suspendido es presentado en la siguiente ecuación.

$\frac{\theta(s)}{V(s)}=\frac{K_{m} / J}{s^{2}+\frac{c}{J} s+\frac{m_{L} \mathrm{~g} d}{J}}=\frac{0.6226}{s^{2}+1.673 s+1.098}$

La respuesta del modelo teórico simulado en matlab se observa en la figura 2.

Figura 2. Respuesta del modelo teórico simulado ante una entrada escalón voltaje en el motor DC aplicada a partir de los $4 \mathrm{~s}$.

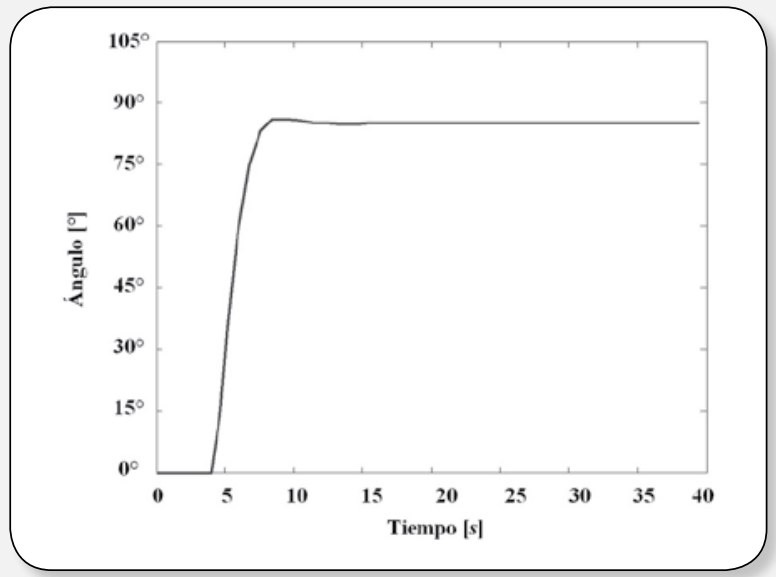

A partir de las características estáticas experimental, de forma práctica y para confirmar el modelo teórico anteriormente obtenido, se identificó el modelo experimental apoyándose en las mediciones de entrada/ salida realizadas al módulo didáctico del péndulo suspendido. En la figura 3, se muestra el comportamiento real del sistema cuando se le aplica al motor DC una entrada escalón en el voltaje alimentación. Dicho comportamiento es representado en una señal de voltaje de retroalimentación entre los 0 hasta $0.6 \mathrm{Vdc}$ que proporciona el potenciómetro correspondiente al comportamiento lineal entre los $0^{\circ}$ hasta $90^{\circ}$ del ángulo entre el brazo oscilante y el eje vertical del péndulo suspendido, como puede ser verificado en la figura 1 .
Figura 3. Respuesta del sistema experimental ante una entrada escalón voltaje en el motor DC
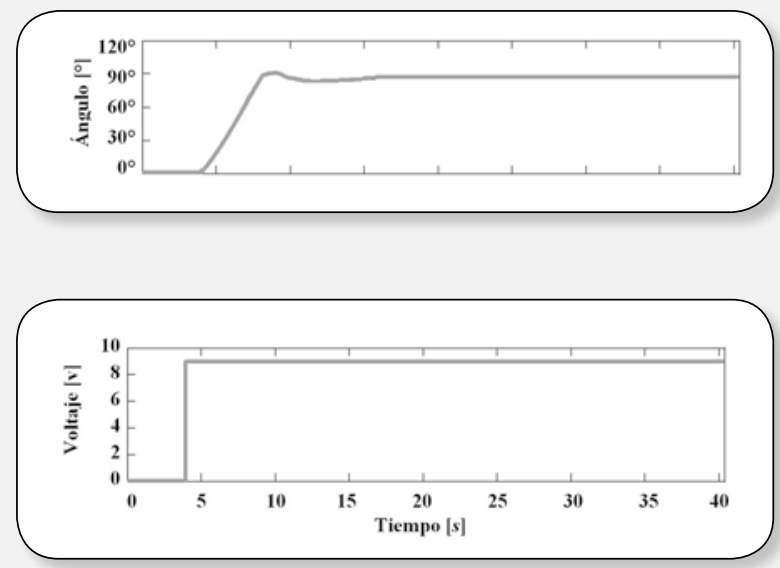

Por medio de la herramienta ident de MATLAB las mediciones de las variables permitieron hacer la respectiva regresión de forma que se determine la función de transferencia que más se ajuste al comportamiento de dicha planta. En la figura 4 pueden apreciarse los diferentes tipos de modelos que se estimaron para analizar con la herramienta ident.

Figura 4. Estimación e correlación de los posibles modelos experimentales a través del ident

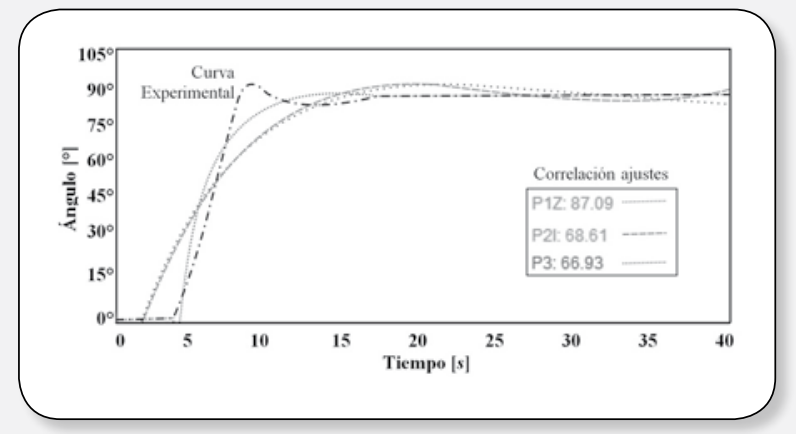

El modelo experimental más adecuado obtenido a través del ident, presentó una correlación del $87.09 \%$ de veracidad. Dicha función de transferencia $\theta(s) / V(s)$ del péndulo suspendido es presentado en la siguiente ecuación.

$$
\frac{\theta(s)}{V(s)}=\frac{0.0647}{s^{2}+0.5 s+0.097}
$$




\section{Respuestas}

\section{Diseño y sintonización del sistema de control}

El sistema péndulo suspendido de la figura 1. fue instrumentado, las variables que están presentes en el sistema son capturadas por un sistema de adquisición de datos a 0,01 [s] de muestreo por un microcontrolador atmega atmel 328 en comunicación serial con la plataforma MATLAB/Simulink, para procesar y monitorear las señales de voltajes que representan las respectivas variables del módulo didáctico. El sistema de adquisición es open-hardware, por lo cual su aplicabilidad es abierta. Es decir, puede utilizarse libremente para el desarrollo de cualquier tipo de proyecto, como observamos en la figura 5 .

El 95\% de los procesos industriales el controlador PID es muy utilizado porque su sintonización es ajustada en campo. Las reglas de sintonía propuesta en la literatura como Ziegler-Nichols pueden permitir ajustes finos en la práctica, [9]. El controlador PID puede ser representado por la siguiente ecuación:

$$
G c(s)=K_{p}\left(1+\frac{1}{T_{i} s}+T_{d} s\right)
$$

Donde $K_{p}$ es la ganancia proporcional, $T_{i}$ es el tiempo integral y $T_{d}$ es el tiempo derivativo.

Por otro lado, como un controlador PID es de fácil aplicación, es posible presentarse algunas limitaciones relacionadas con problemas de sintonía o cambios en el sistema a ser controlado, pero que al final son resueltos por la experiencia del proyectista. Para determinar los parámetros de un controlador que garantice las especificaciones de desempeño, fue aplicado el método de Ziegler e Nichols, sintonizándose un PI (ajuste de los valores $K_{p}$ y $T_{i}$ ) basadas en la respuesta que genera el modelo experimental anteriormente conocido cuando se le aplica una señal escalón unitario, simulando un cambio al voltaje en el motor DC del sistema físico.
Como forma de optimizar la respuesta del controlador PI, fue finamente sintonizados las constantes $K_{b}$ y $T_{i}$ atendiendo el criterio de minimización de índices de desempeño conocido como el integral del valor absoluto del error ponderado en el tiempo (ITAE), $[11,12]$. De forma que nuestro controlador fue finalmente establecido y optimizado representando en la siguiente ecuación de transferencia:

$$
G c(s)=111.27\left(1+\frac{1}{73.55 s}\right)
$$

Figura 5. Lazo de control del sistema péndulo suspendido con un controlador PI desarrollado en Matlab/Simulink.

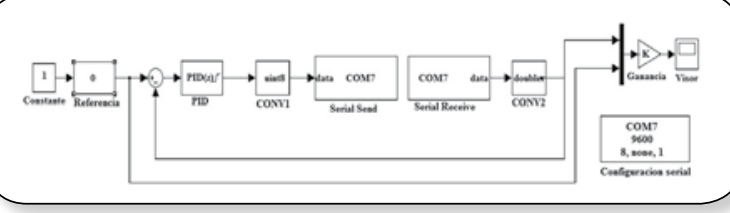

\section{Resultados}

\section{experimentales}

A continuación se presentan algunos resultados de pruebas experimentales utilizando el módulo didáctico péndulo suspendido. Estos resultados satisfacen con las simulaciones obtenidas a través del matlab utilizando el modelo teórico y el modelo experimental.

Para la figura 6 se puede observar la evidencia en la capacidad del controlador en regular el ángulo consigna prestablecido como setpoint. Para esta prueba se hicieron cambios aleatorios en el setpoint, de forma que se observó el efecto del controlador en el sistema para mantener lo más posible el ángulo real al setpoint. En el momento en que se activa el controlador a los $2.3 \mathrm{~s}$, se establece un cambio positivo a $90^{\circ}$ en el setpoint, inmediatamente el controlador PI reacciona y lleva el sistema a alcanzar dicha referencia a los $3.2 \mathrm{~s}$ con un tiempo pico de $0.85 \mathrm{~s}$, con un comportamiento transitorio oscilatorio entre $\operatorname{los} 90^{\circ}$ y $64.5^{\circ}$, 
pero a los $4.4 \mathrm{~s}$ controla por completo los $90^{\circ}$ requeridos; el sistema presenta un tiempo de acomodación de $2.2 \mathrm{~s}$.

A los 9.4 s se le da un cambio negativo y positivo al setpoint referenciadolo a $0^{\circ} \mathrm{e}$ inmediatamente a $90^{\circ}$, se observa en la misma figura 6, como el controlador lleva el sistema en $2.4 \mathrm{~s}$ a estabilizarse finalmente en los $90^{\circ}$.

Figura 6. Comportamiento de la planta bajo la acción del controlador PI a diferentes cambios en el ángulo deseado (setpoint)

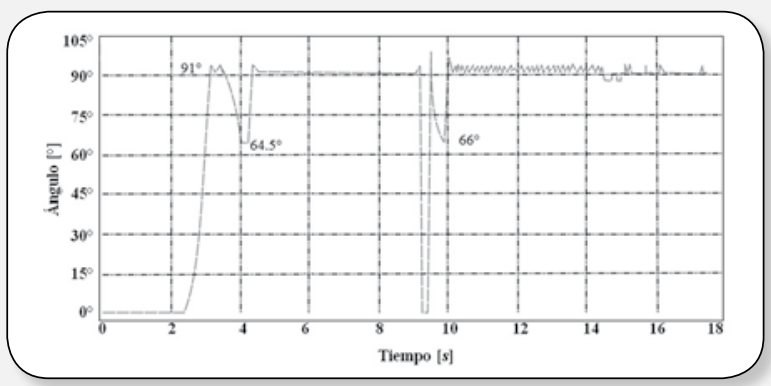

Para la figura 7 , se puede observar el comportamiento del sistema bajo la influencia del controlador PI, manteniendo siempre la consigna de $90^{\circ}$ pero ante perturbaciones inducidas en el brazo del sistema péndulo. La respuesta del sistema de control en malla cerrada es satisfactoria, la perturbación es un impulso (golpe) con dirección vertical negativa del brazo, generando que el potenciómetro de una medida del ángulo muy por debajo al prestablecido en el setpoint $\left(90^{\circ}\right)$. Esta perturbación ocasiona que el controlador actué de forma que cree una respuesta transitorio no mayor a $4 \mathrm{~s}$ para estabilizar el sistema.

Figura 7. Comportamiento de la planta bajo la acción del controlador PI manteniendo su consigna de $90^{\circ}$ deseado (setpoint) ante la presencia de perturbaciones impuestas en el brazo del péndulo.

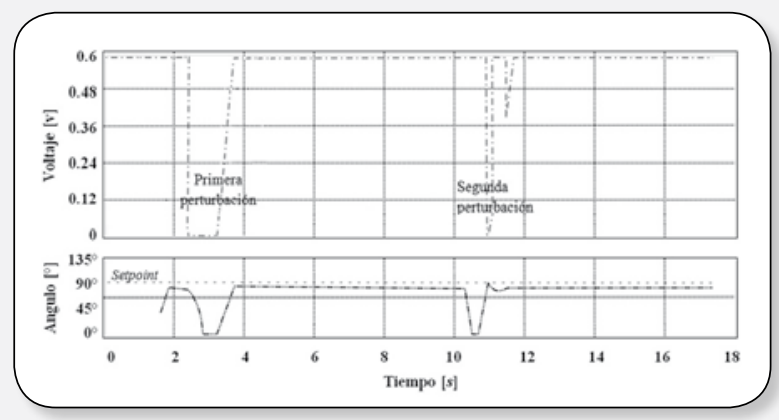

\section{Conclusiones}

Para este proyecto la estructura de control del sistema péndulo suspendido presentó resultados satisfactorios en un margen de controlabilidad para ángulos entre $0^{\circ}$ y $90^{\circ}$ usando el modelo experimental obtenido a través de la herramienta ident de matlab.

Diferentes técnicas de control como por ejemplo lógica fuzzy, redes neurales, algoritmos genéticos podrán ser usadas apoyadas bajo sistema de adquisición de datos gerenciados por microcontroladores en comunicación serial con cualquier plataforma a nivel de software.

Lasherramientas trabajadas enésteproyecto permiten a los estudiantes familiarizarse con todas las etapas del desarrollo de un sistema de control: definición de estudio, modelamiento, sintonización, implementación y comprobación experimental.

Las limitaciones prácticas como máxima tensión aplicada al motor; la sensibilidad y resolución en la lectura del potenciómetro para medir el ángulo real influyeron en los resultados prácticos. Un error en régimen permanente de $5^{\circ}$ que puede ser comprendido como un margen de error tolerable ante la complejidad del manejo y medición de los ángulos.

\section{Referencias Bibliográfias}

ISSN 0122-820X
[1] B. AKTAN, C. BOHUS, L. CROWL, AND M. SHOR, "Distance learning applied to control engineering laboratories," IEEE Transactions on Education, vol. 39, no. 3, pp. 320-326, Aug 1996.

[2] R. A. SERWAY, "Physics: For scientist and engineers with modern physics", Saunders, pp. (468-475, 264-265), Philadelphia, 1990. 
[3] KASRUDDIN, A. N., "Modeling and Controller Design for an Inverted Pendulum System", Master's Thesis, Faculty of Electrical Engineering, Universiti Teknologi Malaysia, April 2007.

[4] HAKAN K., SAADETTIN A., AYDM M. "Sliding mode control of suspended pendulum", Department of electrical and electronics engineering, Sakarya university, Turkey, 2010.

[5] C. EDWARDS, E. F. COLET, L. FRIDMAN, "Advances in variable structure and sliding mode control", 334, Thoma M., Morari M., Springer, pp. 90-120, Berlin, 2006.

[6] KIZIR S., BINGUL Z., OYSU C. "Fuzzy control of a real time inverted pendulum system". Journal of Intelligent \& Fuzzy Systems: Applications in Engineering and Technology, Volume 21 Issue 1, 2 April 2010.

[7] CHARAIS J., LOURENS R, "Software PID Control of an Inverted Pendulum Using the PIC16F684"Microchip Technology Inc. 2004.

[8] HASAN M., SAHA C., RAHMAN M., SARKER R. and ADITYA S. "Balancing of an Inverted Pendulum Using PD Controller". Dhaka Univ. J. Sci. 60(1): 115120, 2012 (January).

[9] OGATA, K, "Ingeniería de Control Moderno". Person Education. $5^{\circ}$ Edición. 2011.

[10] AGUIRRE, L. A. Introdução à Identificação de Sistemas - Técnicas Lineares e Não-lineares Aplicadas a Sistemas Reais. 3 ed. Belo Horizonte, editora UFMG, 2007.

[11] ASTROM, KARL J.; HAGGLUND T. PID controllers: theory, design, and tuning. Second edition. Instrument Society of America. 1995.

[12] ASHAB MIRZA, and CAPT. DR. SARFRAZ HUSSAIN, "Robust Controller for Nonlinear \& Unstable System: Inverted Pendulum", AMSE Journal of Control \& Design Simulation, pp 49-60, Vol. 55, No 3, 4. 2000.

[13] CHATTERJEE D., PATRA A., AND JOGLEKAR H. K., "Swing-up and stabilization of a cart-pendulum system under restricted cart track length," Syst. Control. Lett., vol. 47, no. 11, pp. 355-364, 2002. 